\title{
Preoperative fibrinogen-to-albumin ratio, a potential prognostic factor for patients with stage IB-IIA cervical cancer
}

\author{
Qiang An, Wei Liu, Yujia Yang and Bing Yang*
}

\begin{abstract}
Background: Previous studies have shown that fibrinogen-to-albumin ratio (FAR) is a novel prognostic immune biomarker in various diseases. In this study, we investigated the role of FAR in the prognosis of patients with stage IB-IIA cervical cancer (CC).

Methods: A total of 278 eligible participants with newly diagnosed CC (stage IB-IIA) who had undergone radical hysterectomy followed by adjuvant chemotherapy were enrolled in this study. Demographics, clinicopathological variables, and laboratory tests were obtained from the medical records. Risk factors for overall survival (OS) and recurrence-free survival (RFS) were evaluated by univariate and multivariate Cox proportional regression analyses. The association between OS, RFS, and FAR was assessed by the Kaplan-Meier method using log-rank test.

Results: FAR was associated with age, International Federation of Gynecology and Obstetrics (FIGO) stage, depth of the invasion, and $C$-reactive protein (CRP) level $(P<0.05)$. Preoperative FAR was an effective predictor for OS in CC patients with a cut-off value of 7.75 and an area under the curve (AUC) of $0.707(P<0.001)$. The univariate and multivariate Cox analyses indicated that FIGO stage and FAR were two independent risk factors for both OS and RFS $(P<0.05)$. Kaplan-Meier analysis confirmed that patients with high FAR levels showed significantly lower RFS $(P=0.004)$ and $O S(P=0.003)$ than those with low FAR levels.
\end{abstract}

Conclusions: This study indicated that elevated preoperative FAR might be a novel prognostic factor for CC patients with stage IB-IIA.

Keywords: Cervical cancer, Prognosis, Fibrinogen-to-albumin ratio, Biomarker

\section{Background}

Cervical cancer $(\mathrm{CC})$ is the most common cancer of the female genital tract and the fourth leading cause of malignancy-related deaths among women worldwide [1]. While the incidence and mortality of invasive CC have markedly decreased over time, it still ranks as the third most common cancer in women, with approximately 250,000 patients dying from CC each year globally [2]. Radical hysterectomy followed by chemotherapy or

\footnotetext{
*Correspondence: yangbing_zy@163.com

Department of Gynecology, Affiliated Hospital of Zunyi Medical University, No 149, Dalian road, Zunyi City 563100, Guizhou Province, China
}

chemoradiation has been widely considered as the primary therapeutic strategy for locally advanced CC patients which is defined as International Federation of Gynecology and Obstetrics (FIGO) stage IB-IIA [3]. However, the prognosis is comparatively poor in patients with tumor recurrence due to the limited clinical therapies available [4]. To date, no well-established prognostic biomarkers for CC have been identified. Therefore, a non-invasive, easily accessible pre-treatment biomarker to predict tumor recurrence and prognosis in $\mathrm{CC}$ is urgently required.

Recently, the laboratory indexes including neutrophilto-lymphocyte ratio (NLR) [5], prognostic nutritional 
index (PNI) [6], C-reactive protein (CRP)/albumin ratio (CAR) [7], and albumin-to-alkaline phosphatase ratio (AAPR) [8] have been validated as prognostic factors for CC. In recent decades, systemic inflammation status has served as an important hallmark of malignancy and is closely associated with the initiation, progression, metastasis, as well as resistance to drug therapy [9].

Albumin (Alb) and fibrinogen (Fib) are two commonly used circulating inflammatory proteins. Alb has been reported to be a well-established prognostic factor in patients with various diseases, including oral cavity cancer [10], metastatic pathological femur fractures [11], and amyotrophic lateral sclerosis [12]. Moreover, the prognostic role of Fib has also been reported in various studies, such as spontaneous intracerebral haemorrhage [13] and surgically resected non-small-cell lung cancer [14]. The Fib-to-Alb ratio (FAR), which considers both inflammatory biomarkers, is a novel prognostic immune biomarker in various diseases, e.g. gallbladder cancer [15], breast cancer [16], and ST-segment elevation myocardial infarction [17]. However, whether FAR could be a prognostic factor for $\mathrm{CC}$ has never been examined, which is the main aim of this study.

\section{Methods}

\section{Patients}

This single-center, retrospective study was performed with the approval of the Medical Ethics Committee of our hospital. Eligible participants with newly diagnosed CC admitted to Zunyi Medical University Affiliated Hospital, from June 2010 to December 2017 were enrolled in this study. The inclusion criteria were as follows: (a) newly diagnosed CC patients with the FIGO stage IB-IIA; (b) with clinicopathological and laboratory data including Alb and Fig; (c) who had undergone radical hysterectomy followed by adjuvant chemotherapy; (d) with five-year follow-up data. The exclusion criteria were as follows: (a) with the conditions affecting preoperative Alb or Fib expressions, e.g. infection, inflammation, hematological disease, autoimmune disease, abnormal liver or renal function; (b) combined with other malignancies; (c) without follow-up or complete data. Each enrolled participant was required to submit a signed informed consent.

\section{Treatment schedule}

The diagnosis and treatment schedules of CC patients followed the guidelines by Bhatla et al. [18] All enrolled CC patients had undergone radical hysterectomy and bilateral pelvic lymphadenectomy. Based on the results of postoperative pathology, post-operative platinum-based adjuvant chemotherapy (two or three cycles) with or without concurrent radiotherapy was performed. The pathological definition was carried out by two independent experienced pathologists who were blinded to this study design.

\section{Data collection}

The whole body computed tomography $(\mathrm{CT})$ or positron emission tomography/computed tomography (PET/CT) examination was performed preoperatively. The demographics (including age, body mass index (BMI), and preoperative comorbidities) and clinicopathological variables (including pathological type, FIGO stage, tumor grade, maximum tumor size, adjuvant therapy, lympho-vascular space invasion (LVSI), lymphatic node metastasis (LNM), depth of invasion, and vaginal invasion were extracted from the medical records. Pre-operative laboratory parameters including blood cell analysis (e.g., white blood cell), blood biochemical analysis (e.g., Alb), coagulation analysis (e.g., Fib), and inflammatory cytokines (e.g., CRP) were routinely detected using the blood samples obtained 1 day prior to operation. As described in previous reports, the FAR was calculated by dividing Fib $(\mathrm{mg} / \mathrm{dL})$ by Alb (mg/dL), multiplied by 100 [16].

\section{Prognosis definition}

The clinical and imaging examinations and laboratory tests were assessed for each visit during the follow up. The end point was set as overall survival (OS), recurrence-free survival (RFS), or the due date of followup (December 31, 2017). RFS was defined as the period from the initial surgery to tumor recurrence, death, or the due date. OS was defined as the period from the date of initial surgery to death or the due date.

\section{Statistical analysis}

Statistical analysis was performed using GraphPad prism 8.0 and SPSS 19.0. The predictive and cut-off values of FAR for OS were assessed by receiver operating characteristic (ROC) curve using the Youden index. Chi-square test, Fisher's exact test, Student t-test, or Mann-Whitney U test was performed as appropriate. Risk factors for OS and RFS were evaluated by univariate and multivariate analyses using Cox proportional models. OS and RFS analyses were carried out by Kaplan-Meier method using log-rank test. $P<0.05$ was considered statistically significant.

\section{Results}

\section{Patient characteristics}

A total of 331 CC patients (stage IB-IIA) who had undergone radical hysterectomy were initially enrolled. Among these patients, 53 were excluded (13 with infection or inflammation, 6 with hematological disease, 7 with autoimmune disease, 8 with abnormal liver or renal function, and 19 missing follow-up or complete data) and $278 \mathrm{CC}$ patients were included in the final analysis. According the ROC curve analysis, preoperative FAR 
was an effective predictor for OS in CC patients with a cut-off value of 7.75 and an area under the curve (AUC) of 0.707 (sensitivity: $69.05 \%$, specificity: $70.76 \%, P<$ 0.001 , see Fig. 1) using the Younden index method. Based on the cut-off values, enrolled patients were categorized into high FAR group (FAR $>7.75, n=180,64.7 \%$ ) and low FAR group (FAR $\leq 7.75, n=98,35.3 \%)$. The relationship between clinicopathological characteristics and FAR are listed in Table 1 . The mean age of the whole cohort was 45.5 years and the majority, 89.2\% (248/278), were with squamous cell carcinoma (SCC) patients. Patients with a low FAR were more prone to have a lower age $(P=0.016)$ and a lower FIGO stage $(P=$ 0.033). Those $C C$ patients with a low FAR had a low rate of depth of invasion over $2 / 3(31.1 \%$ vs $44.9 \%, P=0.022)$. No statistical difference was observed between high and low FAR groups with respect to BMI, preoperative comorbidities, pathological type, tumor grade, maximum tumor size, adjuvant therapy, LVSI, LNM, and vaginal invasion $(P>0.05)$. The laboratory tests associated with FAR in CC patients with stage IB-IIA are shown in Table 2. There was no significant difference between patients with high and low FAR except serum CRP concentration $(P=0.012)$.

\section{Risk factors for RFS and OS}

In the total cohort, the 3-year RFS and OS rates were $87.4 \%(243 / 278)$ and $89.9 \%(250 / 278)$, respectively. The 5 -year RFS and OS rates were $79.5 \%(221 / 278)$ and $84.9 \%$ (236/278), respectively. To determine the potential risk factors for RFS and OS, univariate and multivariate Cox proportional regression analyses were performed.
As illustrated by Table 3, FIGO stage (Hazard ratio (HR): 2.11, 95\% confidence interval $(\mathrm{CI}): 1.14-3.79, P=0.017)$, LNM (HR: $1.84,95 \%$ CI: $1.12-2.88, P=0.032$ ) and preoperative FAR level (HR: 2.41, 95\% CI: $1.36-4.11, P=$ 0.011) were three independent risk factors for RFS in CC patients with stage IB-IIA. In addition, FIGO stage (HR: 2.72, 95\% CI: $1.18-5.01, P=0.022)$ and preoperative FAR level (HR: 2.83, 95\% CI: $1.41-5.35, P=0.008$ ) were two independent risk factors for OS in CC patients (Table 4).

\section{PFS and OS associated with FAR}

The results of the Kaplan-Meier analyses confirmed that patients with high FAR level showed significantly lower RFS and OS than those with low FAR by log-rank test $(P=0.004$ in Fig. 2 and $P=0.003$ in Fig. 3, respectively).

\section{Discussion}

Cervical cancer is a significant threat to human health due to its high morbidity and mortality rate. Effective prognostic biomarkers may offer a better option for risk stratification and provide individual appropriate treatment strategies for $\mathrm{CC}$ patients. Parametrial involvement (PMI), positive surgical margins, and LNM are established prognostic factors for tumor recurrence, progression, and clinical outcomes [19]. However, these indexes require an accurate confirmation of postoperative pathology and are time consuming. Therefore investigation to identify an effective, simple, and economic prognostic biomarker for clinical outcomes in CC patients is needed.

In this study, we demonstrated that FAR was an independent prognostic indicator for $\mathrm{CC}$ patients with stage IB-IIA. To our knowledge, this was the first study

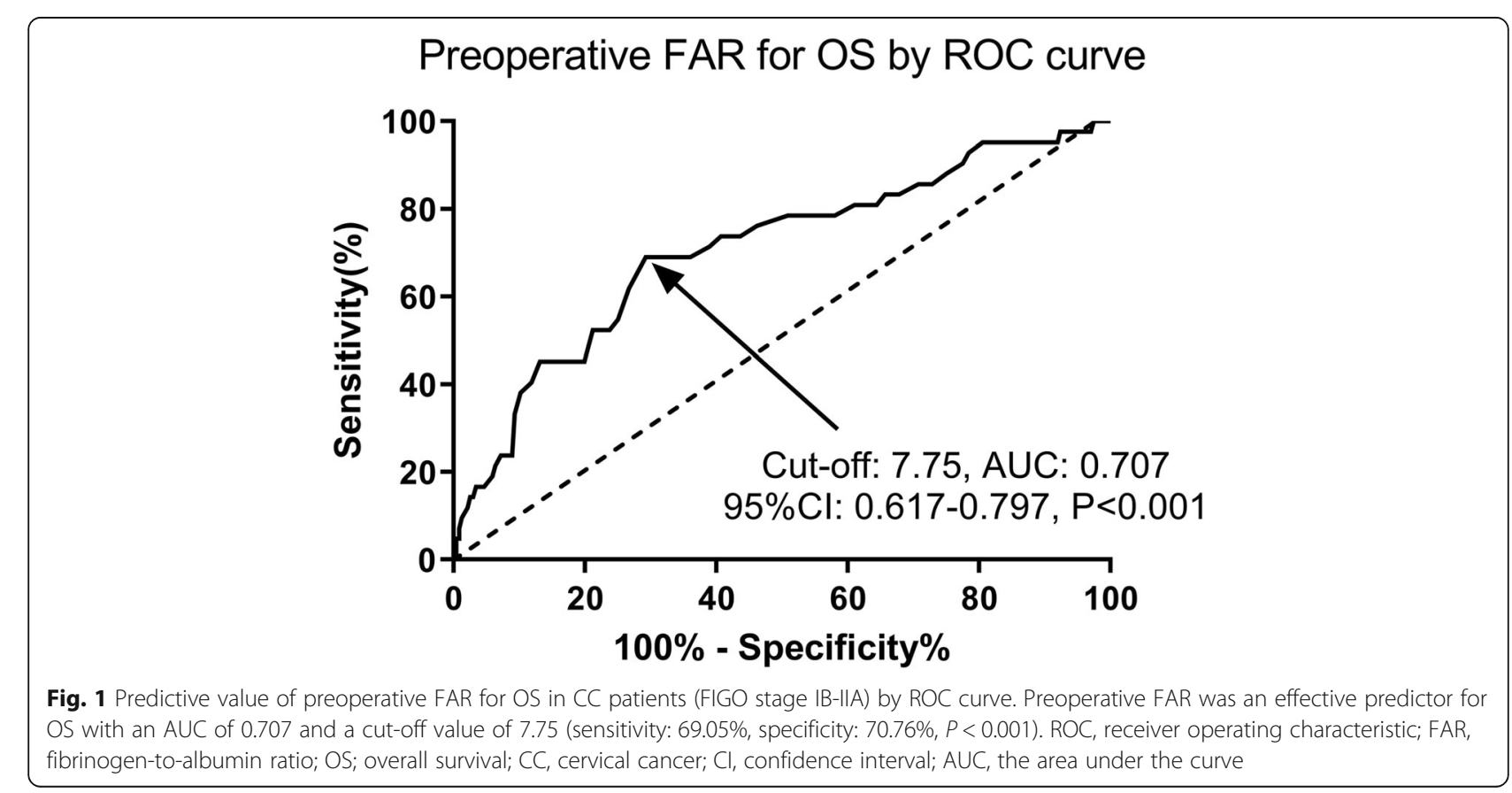


Table 1 Clinicopathological variables associated with FAR in CC patients with stage IB-IIA

\begin{tabular}{|c|c|c|c|c|}
\hline Parameters & Total & $F A R \leq 7.75$ & FAR $>7.75$ & $p$ value \\
\hline Number & 278 & 180 & 98 & - \\
\hline Age (years) & $45.5 \pm 6.3$ & $44.8 \pm 6.7$ & $46.8 \pm 6.4$ & $0.016^{*}$ \\
\hline BMI $\left(\mathrm{kg} / \mathrm{m}^{2}\right)$ & $22.3 \pm 2.0$ & $22.3 \pm 2.1$ & $22.4 \pm 1.8$ & 0.691 \\
\hline Comorbidities (n, \%) & & & & - \\
\hline Diabetes & $31(11.2)$ & $20(11.1)$ & $11(11.2)$ & 0.977 \\
\hline Hypertension & $39(14.0)$ & $22(12.2)$ & $17(17.3)$ & 0.240 \\
\hline Hyperlipemia & $26(9.4)$ & $17(9.4)$ & $9(9.2)$ & 0.943 \\
\hline Pathological type & & - & - & 0.297 \\
\hline SCC & $248(89.2)$ & $158(87.7)$ & $90(91.8)$ & - \\
\hline Non-SCC & $30(10.8)$ & $22(12.3)$ & $8(8.2)$ & - \\
\hline FIGO stage & & - & - & $0.033^{*}$ \\
\hline IB & $146(50.4)$ & $103(57.2)$ & $43(43.9)$ & - \\
\hline$\| \mathrm{A}$ & $132(47.5)$ & $77(42.8)$ & $55(56.1)$ & - \\
\hline Tumor grade (n, \%) & & - & - & 0.590 \\
\hline G1 & $53(19.1)$ & $36(20.0)$ & $17(17.3)$ & - \\
\hline G2 & $166(59.7)$ & $109(60.6)$ & $57(58.2)$ & - \\
\hline G3 & $59(21.2)$ & $35(19.4)$ & $24(24.5)$ & - \\
\hline Maximum tumor size & & - & - & 0.447 \\
\hline$\geq 4 \mathrm{~cm}$ & 99 (35.6) & 67 (37.2) & $32(32.7)$ & - \\
\hline$<4 \mathrm{~cm}$ & $179(64.4)$ & $113(62.8)$ & $66(67.3)$ & - \\
\hline Adjuvant therapy & & & & 0.748 \\
\hline No & $54(19.4)$ & $42(23.3)$ & $12(12.2)$ & - \\
\hline Chemoradiotherapy & $77(27.7)$ & $45(25.0)$ & $32(32.7)$ & - \\
\hline Chemotherapy & 108 (38.8) & $67(37.2)$ & $41(41.8)$ & - \\
\hline Radiotherapy & $39(10.8)$ & $26(14.4)$ & $13(7.2)$ & - \\
\hline LVSI & & - & - & 0.409 \\
\hline No & $247(88.8)$ & $162(90.0)$ & $85(86.7)$ & - \\
\hline Yes & $31(11.2)$ & $18(10.0)$ & $13(13.3)$ & - \\
\hline LNM & & & & 0.547 \\
\hline No & $232(83.5)$ & $152(84.4)$ & 80 (81.6) & - \\
\hline Yes & $46(16.5)$ & $28(15.6)$ & $18(18.4)$ & - \\
\hline Depth of invasion & & - & - & $0.022^{*}$ \\
\hline$\geq 2 / 3$ & $100(36.0)$ & $56(31.1)$ & $44(44.9)$ & - \\
\hline$<2 / 3$ & $178(64.0)$ & $124(68.9)$ & $54(55.1)$ & - \\
\hline Vaginal invasion & & - & - & 0.738 \\
\hline No & $260(93.5)$ & $169(93.9)$ & $91(92.9)$ & - \\
\hline Yes & $18(6.5)$ & $11(6.1)$ & $7(7.1)$ & - \\
\hline
\end{tabular}

Abbreviations: CC cervical cancer, BMI body mass index, SCC squamous cell carcinoma, FIGO International Federation of Gynecology and Obstetrics, FAR fibrinogento-albumin ratio, LVSI lympho-vascular space invasion, LNM lymphatic node metastasis $P$-values were calculated by Student's $t$ test, Mann-Whitney $U$ test or Chi-squared test * $P<0.05$

concerning the prognostic implication of FAR in CC. Prognostic role of serum Alb and Fib has been reported in various studies. Taken together, the prognostic role of FAR was far superior to either Alb or Fib alone. In our study, FAR was observed to be associated with some clinicopathological indicators (i.e., age, FIGO stage, and depth of invasion), indicating a potential close correlation between FAR level and the aggressiveness and progression of CC. Fib is a key factor in the coagulation system [20], while Alb is an objective indicator reflecting the 
Table 2 Laboratory tests associated with FAR in CC patients with stage IB-IIA

\begin{tabular}{lllll}
\hline Laboratory tests & Total & FAR $\leq 7.75$ & FAR> 7.75 & $p$ value \\
\hline Number & 278 & 180 & 98 & - \\
Hemoglobin $(\mathrm{g} / \mathrm{L})$ & $104.9 \pm 10.5$ & $105.3 \pm 10.2$ & $104.3 \pm 11.2$ & $168.5 \pm 36.3$ \\
Platelet $\left(10^{9} / \mathrm{L}\right)$ & $171.9 \pm 37.1$ & $173.8 \pm 40.5$ & $7.1 \pm 1.7$ & 0.281 \\
$\mathrm{WBC}\left(10^{9} / \mathrm{L}\right)$ & $7.0 \pm 1.9$ & $7.0 \pm 2.1$ & $4.2 \pm 0.7$ & 0.686 \\
Total cholesterol & $4.3 \pm 0.8$ & $4.3 \pm 0.8$ & & 0.300 \\
$(\mathrm{mmol} / \mathrm{L})$ & & & $7.7 \pm 9.5$ & $0.012^{*}$ \\
CRP $(\mathrm{mg} / \mathrm{L})$ & $6.1 \pm 7.9$ & $5.2 \pm 6.9$ &
\end{tabular}

Abbreviations: WBC white blood cell, CRP C-reactive protein, CC cervical cancer, FAR fibrinogen-to-albumin ratio

$P$-values were calculated by Student's t test or Mann-Whitney $U$ test

* $P<0.05$

nutritional condition [21]. Increased Fib level is widely observed in patients with solid malignancies and is closely associated with the size, deep stromal invasion, progression, and recurrence of tumors [22, 23]. Meanwhile, there is increasing evidence that Alb may be used for the early diagnosis, prognosis, or prediction of solid malignancies [24]. A recent study by Seebacher et al. has indicated Alb as part of a prognostic model regarding recurrent CC [25].

Moreover, Fib and Alb are widely accepted as two acute phase response proteins for the systemic inflammatory status [26]. Fib and Alb are both synthesized by hepatocytes and they vary oppositely under inflammatory stimulation [27]. Taken together, FAR is a critical factor in nutrition condition, coagulation system, and systemic inflammation. Furthermore, these processes were all closely associated with the survival, intravasation, and adhesion of tumor cells, leading to increased metastatic potential [28], which might be a possible explanation for the prognostic role of FAR in CC patients. Furthermore, a recent study by Huang et al. has indicated that pretreatment Alb/Fib

Table 3 Risk factors for RFS in CC patients with stage IB-IIA by univariate and multiple Cox regression analysis

\begin{tabular}{|c|c|c|c|c|}
\hline \multirow[t]{2}{*}{ Variables } & \multicolumn{4}{|c|}{ Univariate Multivariate } \\
\hline & $\mathrm{HR}(95 \% \mathrm{Cl})$ & $p$ value & $\mathrm{HR}(95 \% \mathrm{Cl})$ & $p$ value \\
\hline Age (high vs low) & $1.47(0.97-2.21)$ & 0.076 & & \\
\hline BMI (high vs low) & $0.99(0.67-1.48)$ & 0.923 & & \\
\hline Diabetes (yes vs no) & $1.21(0.82-1.77)$ & 0.358 & & \\
\hline Hypertension (yes vs no) & $1.03(0.63-1.65)$ & 0.892 & & \\
\hline Hyperlipemia (yes vs no) & $1.11(0.72-1.61)$ & 0.628 & & \\
\hline Pathological type (SCC vs non-SCC) & $1.21(0.30-4.93)$ & 0.783 & & \\
\hline FIGO stage (IIA vs IB) & $2.33(1.29-4.24)$ & $0.009^{*}$ & $2.11(1.14-3.79)$ & $0.017^{*}$ \\
\hline Tumor grade (G2/3 vs G1) & $1.16(0.68-2.06)$ & 0.553 & & \\
\hline Tumor size $(\geq 4 \mathrm{~cm}$ vs $<4 \mathrm{~cm}$ ) & $1.83(1.26-4.27)$ & $0.026^{*}$ & $1.57(0.88-2.81)$ & 0.136 \\
\hline Adjuvant therapy (yes vs no) & $1.61(0.47-5.11)$ & 0.413 & & \\
\hline LVSI (yes vs no) & $1.09(0.69-1.69)$ & 0.676 & & \\
\hline LNM (yes vs no) & $1.59(1.04-2.49)$ & $0.029^{*}$ & $1.84(1.12-2.88)$ & $0.032^{*}$ \\
\hline Depth of invasion $(\geq 2 / 3$ vs $<2 / 3)$ & $1.16(0.46-2.92)$ & 0.718 & & \\
\hline Vaginal invasion (yes vs no) & $1.24(0.70-2.21)$ & 0.411 & & \\
\hline Hemoglobin (high vs low) & $0.74(0.31-1.79)$ & 0.521 & & \\
\hline Platelet (high vs low) & $1.11(0.60-2.04)$ & 0.712 & & \\
\hline WBC (high vs low) & $1.66(0.52-5.22)$ & 0.388 & & \\
\hline Total cholesterol (high vs low) & $1.14(0.73-1.82)$ & 0.554 & & \\
\hline CRP (high vs low) & $1.32(0.49-3.14)$ & 0.533 & & \\
\hline FAR $(>7.75$ vs $\leq 7.75)$ & $2.53(1.24-5.18)$ & $0.009^{*}$ & $2.41(1.36-4.11)$ & $0.011^{*}$ \\
\hline
\end{tabular}

Abbreviations: CC cervical cancer, BMI body mass index, SCC squamous cell carcinoma, FIGO International Federation of Gynecology and Obstetrics, FAR fibrinogento-albumin ratio, LVSI lympho-vascular space invasion, LNM lymphatic node metastasis, WBC white blood cell, CRP C-reactive protein, $H R$ hazard ratio, $C I$ confidence interval

${ }^{*} P<0.05$ 
Table 4 Risk factors for OS in CC patients with stage IB-IIA by univariate and multiple Cox regression analysis

\begin{tabular}{|c|c|c|c|c|}
\hline \multirow[t]{2}{*}{ Variables } & \multicolumn{4}{|c|}{ Univariate Multivariate } \\
\hline & $\mathrm{HR}(95 \% \mathrm{Cl})$ & $p$ value & $\mathrm{HR}(95 \% \mathrm{Cl})$ & $p$ value \\
\hline Age (high vs low) & $1.74(0.88-3.51)$ & 0.113 & & \\
\hline BMI (high vs low) & $1.11(0.56-2.14)$ & 0.751 & & \\
\hline Diabetes (yes vs no) & $1.08(0.58-2.02)$ & 0.765 & & \\
\hline Hypertension (yes vs no) & $1.15(0.61-2.11)$ & 0.651 & & \\
\hline Hyperlipemia (yes vs no) & $1.43(0.62-3.22)$ & 0.357 & & \\
\hline Pathological type (SCC vs non-SCC) & $1.89(0.92-4.11)$ & 0.079 & & \\
\hline FIGO stage (IIA vs IB) & $2.91(1.32-6.14)$ & $0.008^{*}$ & $2.72(1.18-5.01)$ & $0.022^{*}$ \\
\hline Tumor grade (G2/3 vs G1) & $0.98(0.50-1.87)$ & 0.914 & & \\
\hline Tumor size ( $\geq 4 \mathrm{~cm}$ vs $<4 \mathrm{~cm}$ ) & $1.41(0.56-3.31)$ & 0.431 & & \\
\hline Adjuvant therapy (yes vs no) & $1.59(0.93-2.68)$ & 0.087 & & \\
\hline LVSI (yes vs no) & $2.53(1.20-5.61)$ & $0.016^{*}$ & $1.48(0.81-2.69)$ & 0.211 \\
\hline LNM (yes vs no) & $1.84(0.67-4.79)$ & 0.211 & & \\
\hline Depth of invasion( $\geq 2 / 3$ vs < 2/3) & $1.41(1.07-1.87)$ & $0.018^{*}$ & $1.22(0.83-1.77)$ & 0.281 \\
\hline Vaginal invasion (yes vs no) & $1.33(0.96-1.78)$ & 0.081 & & \\
\hline Hemoglobin (high vs low) & $0.70(0.40-1.24)$ & 0.265 & & \\
\hline Platelet (high vs low) & $1.55(0.97-2.47)$ & 0.061 & & \\
\hline WBC (high vs low) & $1.44(0.95-2.11)$ & 0.068 & & \\
\hline Total cholesterol (high vs low) & $1.26(0.65-2.33)$ & 0.443 & & \\
\hline CRP (high vs low) & $1.25(0.84-1.81)$ & 0.194 & & \\
\hline FAR $(>7.75$ vs $\leq 7.75)$ & $2.78(1.56-5.06)$ & $0.003^{*}$ & $2.83(1.41-5.35)$ & $0.008^{*}$ \\
\hline
\end{tabular}

Abbreviations: CC cervical cancer, BMI body mass index, SCC squamous cell carcinoma, FIGO International Federation of Gynecology and Obstetrics, FAR fibrinogento-albumin ratio, LVSI lympho-vascular space invasion, LNM lymphatic node metastasis, WBC white blood cell, CRP C-reactive protein, $H R$ hazard ratio, CI confidence interval

$* P<0.05$

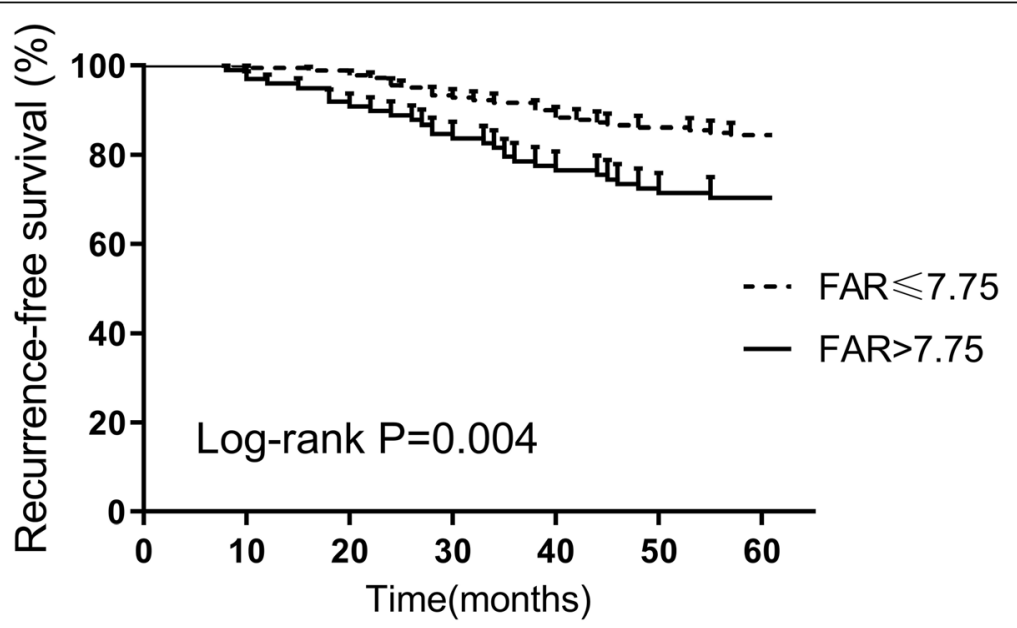

Fig. 2 Recurrence-free survival in CC patients and preoperative FAR by Kaplan-Meier curve analysis. A higher preoperative FAR (> 7.75) correlated with a worse recurrence-free survival $(P=0.004)$. FAR, fibrinogen-to-albumin ratio; $C C$, cervical cancer 


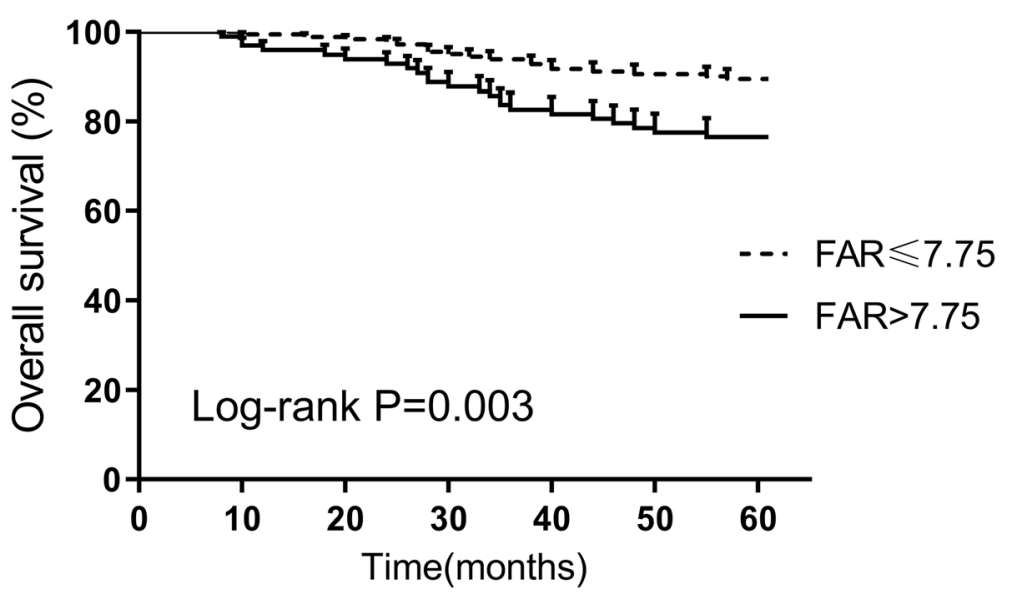

Fig. 3 Overall survival in CC patients and preoperative FAR by Kaplan-Meier curve analysis. A higher preoperative FAR (> 7.75) correlated with a worse overall survival $(P=0.003)$. FAR, fibrinogen-to-albumin ratio; $C C$, cervical cancer

ratio could improve the diagnostic efficiency of $\mathrm{CC}$ alone or combined with tumor biomarkers [24].

The prognostic role of FAR has been reported for patients with malignancies in recent studies, including esophageal squamous cell carcinoma [29], breast cancer [16], metastatic colorectal cancer [30], hepatocellular carcinoma [31], and gallbladder cancer [15]. In addition, a recent study by Liu et al. indicates that FAR may act as a novel inflammatory parameter reflecting disease activity of ankylosing spondylitis [27]. The prognostic role of FAR is also observed in patients with non-ST elevation acute coronary syndrome after percutaneous coronary intervention [32]. A recent meta-analysis by Zhang et al. has uncovered the close correlation between FAR and positive lymph node metastasis, distant metastasis, deeper infiltration, and advanced clinical stage in human malignant tumors [33]. Similarly, a recent study by $\mathrm{Yu}$ et al. indicates preoperative albumin-to-fibrinogen ratio as an independent predictor for chemotherapy resistance and prognosis in advanced epithelial ovarian cancer [34]. All these studies are in line with our results. Furthermore, the optimal cut-off values of FAR for prognosis vary in different reports with unknown underlying mechanisms. The different biological behaviors of tumors, sample sizes, cohort characteristics, racial differences, and population heterogeneity might be potential explanations for the inconsistent findings. Therefore, more large-scale studies are urgently needed to verify the conclusions. However, we have to admit that the study findings and conclusions would be more robust if a validation cohort was used.

\section{Conclusions}

In conclusion, this study indicated that elevated preoperative FAR might be a novel prognostic factor for CC patients with FIGO stage IB-IIA.
Abbreviations

CC: Cervical cancer; SCC: Squamous cell carcinoma; BMI: Body mass index; FIGO: International Federation of Gynecology and Obstetrics; LVSI: Lymphovascular space invasion; LNM: Lymphatic node metastasis; FAR: Fibrinogento-albumin ratio; WBC: White blood cell; CRP: C-reactive protein; RFS: Recurrence-free survival; OS: Overall survival; ROC: Receiver operating characteristic; AUC: Area under the curve; HR: Hazard ratio; Cl: Confidence interval

\section{Acknowledgments \\ None.}

\section{Authors' contributions}

QA: Project development, Data collection, Data analysis, Manuscript writing WL: Data collection. YJ Y: Project development. B Y: Project development and data analysis. All authors have read and approved the manuscript.

Funding

None.

Availability of data and materials

Please contact the author Bing Yang (yangbing_zy@163.com) upon reasonable requests.

\section{Ethics approval and consent to participate}

This study was performed with the approval of the Medical Ethics Committee of Affiliated Hospital of Zunyi Medical University. The patients enrolled all presented written informed consent.

\section{Consent for publication}

Not applicable.

\section{Competing interests}

All the authors declare that they have no competing interests.

Received: 8 May 2020 Accepted: 16 July 2020

Published online: 25 July 2020

References

1. Torre LA, Bray F, Siegel RL, Ferlay J, Lortet-Tieulent J, Jemal A. Global cancer statistics, 2012. CA Cancer J Clin. 2015;65(2):87-108.

2. Siegel RL, Miller KD, Jemal A. Cancer statistics, 2017. CA Cancer J Clin. 2017; 67(1):7-30.

3. Koh WJ, Abu-Rustum NR, Bean S, Bradley K, Campos SM, Cho KR, Chon HS, Chu C, Clark R, Cohn D, et al. Cervical Cancer, version 3.2019, NCCN clinical practice guidelines in oncology. J Natl Compr Cancer Netw. 2019;17(1):64-84. 
4. Kim MK, Jo H, Kong HJ, Kim HC, Kim JW, Kim YM, Song YS, Kang SB, Mok JE, Lee HP. Postoperative nomogram predicting risk of recurrence after radical hysterectomy for early-stage cervical cancer. Int J Gynecol Cancer. 2010; 20(9):1581-6.

5. Dolan RD, McSorley ST, Horgan PG, Laird B, McMillan DC. The role of the systemic inflammatory response in predicting outcomes in patients with advanced inoperable cancer: systematic review and meta-analysis. Crit Rev Oncol Hematol. 2017:116:134-46.

6. Haraga J, Nakamura K, Omichi C, Nishida T, Haruma T, Kusumoto T, Seki N, Masuyama H, Katayama N, Kanazawa S, et al. Pretreatment prognostic nutritional index is a significant predictor of prognosis in patients with cervical cancer treated with concurrent chemoradiotherapy. Mol Clin Oncol. 2016;5(5):567-74.

7. Zhang W, Liu K, Ye B, Liang W, Ren Y. Pretreatment C-reactive protein/ albumin ratio is associated with poor survival in patients with stage IB-IIA cervical cancer. Cancer Med. 2018;7(1):105-13.

8. Z Zhang C, Li Y, Ji R, Zhang W, Zhang C, Dan Y, Qian H, He A. The prognostic significance of pretreatment albumin/alkaline phosphatase ratio in patients with stage IB-IIA cervical Cancer. Onco Targets Ther. 2019;12:9559-68.

9. Mantovani A, Allavena P, Sica A, Balkwill F. Cancer-related inflammation. Nature. 2008:454(7203):436-44.

10. Valero C, Zanoni DK, Pillai A, Ganly I, Morris LGT, Shah JP, Wong RJ, Patel SG. Host Factors Independently Associated With Prognosis in Patients With Oral Cavity Cancer. JAMA Otolaryngol Head Neck Surg. 2020.

11. Sim DS, Zainul-Abidin S, Sim EY, Seng CS, Tan SE, Tan MH, Howe TS, Koh JSB. Serum albumin level predicts survival after surgical treatment of metastatic femur fractures: a retrospective study. J Orthop Surg Res. 2020;15(1):128.

12. Sun J, Carrero JJ, Zagai U, Evans M, Ingre C, Pawitan Y, Fang F. Blood biomarkers and prognosis of amyotrophic lateral sclerosis. Eur J Neurol. 2020

13. Song X, Zhang Q, Cao Y, Wang S, Zhao J. Antiplatelet therapy does not increase mortality of surgical treatment for spontaneous intracerebral haemorrhage. Clin Neurol Neurosurg. 2020;196:105873.

14. Ohara S, Suda K, Tomizawa K, Takemoto T, Fujino T, Hamada A, Koga T, Nishino M, Chiba M, Sato K, et al. Prognostic value of plasma fibrinogen and D-dimer levels in patients with surgically resected non-small cell lung cancer. Surg Today. 2020.

15. Xu WY, Zhang HH, Xiong JP, Yang XB, Bai Y, Lin JZ, Long JY, Zheng YC, Zhao $H T$, Sang XT. Prognostic significance of the fibrinogen-to-albumin ratio in gallbladder cancer patients. World J Gastroenterol. 2018;24(29):3281-92.

16. Hwang KT, Chung JK, Roh EY, Kim J, Oh S, Kim YA, Rhu J, Kim S. Prognostic influence of preoperative fibrinogen to albumin ratio for breast Cancer. J Breast Cancer. 2017;20(3):254-63.

17. Zhao Y, Yang J, Ji Y, Wang S, Wang T, Wang F, Tang J. Usefulness of fibrinogen-to-albumin ratio to predict no-reflow and short-term prognosis in patients with ST-segment elevation myocardial infarction undergoing primary percutaneous coronary intervention. Heart Vessel. 2019;34(10):1600-7.

18. Bhatla N, Aoki D, Sharma DN, Sankaranarayanan R. Cancer of the cervix uteri. Int J Gynaecol Obstet. 2018;143(Suppl 2):22-36.

19. Canaz E, Ozyurek ES, Erdem B, Aldikactioglu Talmac M, Yildiz Ozaydin I, Akbayir O, Numanoglu C, Ulker V. Preoperatively assessable clinical and pathological risk factors for Parametrial involvement in surgically treated FIGO stage IB-IIA cervical Cancer. Int J Gynecol Cancer. 2017;27(8):1722-8.

20. Chapin JC, Hajjar KA. Fibrinolysis and the control of blood coagulation Blood Rev. 2015;29(1):17-24.

21. Keller U. Nutritional Laboratory Markers in Malnutrition. J Clin Med. 2019;8(6): 775.

22. Luo Y, Kim HS, Kim M, Lee M, Song YS. Elevated plasma fibrinogen levels and prognosis of epithelial ovarian cancer: a cohort study and metaanalysis. J Gynecol Oncol. 2017;28(3):e36.

23. Zhang X, Long Q. Elevated serum plasma fibrinogen is associated with advanced tumor stage and poor survival in hepatocellular carcinoma patients. Medicine (Baltimore). 2017;96(17):e6694.

24. Huang L, Mo Z, Zhang L, Qin S, Qin S, Li S. Diagnostic value of albumin to fibrinogen ratio in cervical Cancer. Int J Biol Markers. 2020; 1724600820915916.

25. Seebacher V, Sturdza A, Bergmeister B, Polterauer S, Grimm C, Reinthaller A, Hilal Z, Aust S. Factors associated with post-relapse survival in patients with recurrent cervical cancer: the value of the inflammation-based Glasgow prognostic score. Arch Gynecol Obstet. 2019;299(4):1055-62.

26. Luyendyk JP, Schoenecker JG, Flick MJ. The multifaceted role of fibrinogen in tissue injury and inflammation. Blood. 2019;133(6):511-20.
27. Liu M, Huang Y, Huang Z, Zhong Z, Deng W, Huang Z, Huang Q, Li T. The role of fibrinogen to albumin ratio in ankylosing spondylitis: correlation with disease activity. Clin Chim Acta. 2020;505:136-40.

28. Palumbo JS, Kombrinck KW, Drew AF, Grimes TS, Kiser JH, Degen JL, Bugge $\mathrm{TH}$. Fibrinogen is an important determinant of the metastatic potential of circulating tumor cells. Blood. 2000;96(10):3302-9.

29. Tan Z, Zhang M, Han Q, Wen J, Luo K, Lin P, Zhang L, Yang H, Fu J. A novel blood tool of cancer prognosis in esophageal squamous cell carcinoma: the fibrinogen/albumin ratio. J Cancer. 2017;8(6):1025-9.

30. Zhang L, Zhang J, Wang Y, Dong Q, Piao H, Wang Q, Zhou Y, Ding Y. Potential prognostic factors for predicting the chemotherapeutic outcomes and prognosis of patients with metastatic colorectal cancer. J Clin Lab Anal. 2019;33(8):e22958.

31. Xu Q, Yan Y, Gu S, Mao K, Zhang J, Huang P, Zhou Z, Chen Z, Zheng S, Liang J, et al. A novel inflammation-based prognostic score: the fibrinogen/ albumin ratio predicts prognoses of patients after curative resection for hepatocellular carcinoma. J Immunol Res. 2018;2018:4925498.

32. He D, Jiao Y, Yu T, Song J, Wen Z, Wu J, Duan W, Sun N, Sun Z, Sun Z Prognostic value of fibrinogen-to-albumin ratio in predicting 1-year clinical progression in patients with non-ST elevation acute coronary syndrome undergoing percutaneous coronary intervention. Exp Ther Med. 2019;18(4): 2972-8

33. Zhang Y, Xiao G. Prognostic significance of the ratio of fibrinogen and albumin in human malignancies: a meta-analysis. Cancer Manag Res. 2019; 11:3381-93

34. Yu W, Ye Z, Fang $X$, Jiang $X$, Jiang $Y$. Preoperative albumin-to-fibrinogen ratio predicts chemotherapy resistance and prognosis in patients with advanced epithelial ovarian cancer. J Ovarian Res. 2019;12(1):88.

\section{Publisher's Note}

Springer Nature remains neutral with regard to jurisdictional claims in published maps and institutional affiliations.

Ready to submit your research? Choose BMC and benefit from:

- fast, convenient online submission

- thorough peer review by experienced researchers in your field

- rapid publication on acceptance

- support for research data, including large and complex data types

- gold Open Access which fosters wider collaboration and increased citations

- maximum visibility for your research: over $100 \mathrm{M}$ website views per year

At $\mathrm{BMC}$, research is always in progress.

Learn more biomedcentral.com/submissions 